\title{
Implementation of CWDM technique in access network
}

\author{
N. Ali ${ }^{1,2,4^{*}}$, S. Shaari ${ }^{1}, N$. A. M. Ahmad Hambali1 ${ }^{1,2}$, Liza M. A ${ }^{3}$ and C. B. M. Rashidi ${ }^{4}$ \\ ${ }^{1}$ School of Microelectronics Engineering, Universiti Malaysia Perlis (UniMAP), 02600 Arau, Perlis, Malaysia \\ ${ }^{2}$ Semiconductor Photonics \& Integrated Lightwave Systems (SPILS), School of Microelectronic Engineering, Universiti Malaysia \\ Perlis, Pauh Putra Main Campus, 02600 Arau, Perlis, Malaysia \\ ${ }^{3}$ University Kuala Lumpur British Malaysian Institute, Batu 8, Jalan Sungai Pusu 53100, Gombak Selangor \\ ${ }^{4}$ Advanced Communication Engineering, Centre of Excellence-School of Computer and Communication Engineering, Universiti \\ Malaysia Perlis
}

\begin{abstract}
The Coarse Wavelength Division Multiplexing (CWDM) system presented in this paper has several distinct characteristics from the standard CWDM system. One of them is the channel spacing whereby it uses smaller channel spacing compared to the standard system. The other is that, the system uses a broad spectrum laser source. The source is filtered by using 'Bragg Grating' before being transmitted. The filtration gives higher OSNR compared to standard CWDM setup. It was found that the system can transmit through each channel up to $2.5 \mathrm{Gbps}$ with the probability of error of $10^{-12}$. The maximum distance that can be supported by this system is about $27 \mathrm{~km}$, for the data rate of $155 \mathrm{Mbps}$.
\end{abstract}

\section{Introduction}

Access network mainly comprises of the voice network, which is traditionally based on copper twisted pair cable. This is still a viable option to a certain extend in the network today even if the data loading in the network increases since the technology in communication system is also improving. As the speed increases further, copper cables can no longer be used and need to be replaced by fiber cables as feeder line to the copper cables, which are then connected to the customer. This is the reason that in the latest trend [1] the link between telco exchanges and the customer is connected all the way by fiber. In existing architecture the access distributions are using copper cable with coverage of $4 \mathrm{~km}$ in radii. The coverage span limits the connection from the access node to the end user. The optical connection is used when the coverage of copper cable cannot be supported and a high bandwidth line is needed. Optical lines will increased the price of service but by providing high bandwidth to the end user the price will be economical. The problem arises in the optical lines transmission where, the lines are not shareable by the other users in order to connect to medium and lower class users. The low-bandwidth users that are far away from the access node; i.e. unreachable by the copper cable; cannot be connected. One of the solutions available is FTTx system. FTTx is beneficial because it can support not only low bandwidth but also high bandwidth and long distance. However, the cost incurred by FTTx is prohibitive and not practical for medium-bandwidth users. One of them is FTTH (Fiber to the Home)

\footnotetext{
* Corresponding author: norshamsuri@unimap.edu.my
}

Many studies have been carried out to reduce the cost of FTTx i.e. FTTH system such as Yaojun et. al. [2]. The architecture proposed to maximize the utilization of the optical lines in order to reduce the cost of implementation. The CWDM implementation in this architecture is only available at the feeder of access network, and it does not cover up to the distribution of access network. The distributions use FTTx system for higher end user and copper to lower end user. The architecture does not cover medium bandwidth user and also the lower bandwidth user, which are located far away from the access node. Even though the utilization of the access node using CWDM can reduce the cost but it still cannot support the optical lines for medium and lower bandwidth user. Most of the time the connection to the end user using optical lines is dedicated for high end user only. In this architecture, the connection to the end user has to use a new scheme since the network architecture cannot support existing scheme or data type. The author proposed modulator on each user, which increased the cost of implementation. In the architecture proposed, the distribution of the channel uses power splitting which reduces the cost but it has to place erbium-doped amplifier (EDFA) to increase the power reduction during splitting which increases the level of noise and also raises the cost of system. The wavelength spacing used is $3.6 \mathrm{~nm}$, which is larger compared to our architecture (2 $\mathrm{nm})$.

Our architecture network has a new multiplexing device that can integrate the two physical types of transmitted data: optical and electrical. This device will 
work on the cascaded network architecture where the access network will consist of an optical network and a copper network. The system can transmit up to $2.5 \mathrm{Gbps}$ on each channel with probability of errors of $10^{-12}$. The existing scheme can be integrated into the architecture since the system is transparent of data format.

\section{WORKING PRINCIPLE AND SYSTEM DESCRIPTION}

In the existing architecture users are connected by the access distribution network. The network normally starts from the exchange terminal and in urban areas they are connected from the curb nearby. The network architecture is normally in point-to-point form where the connection is done directly to access node. The connections are implemented using a copper cable and for high-end lines they use optical lines. Most paper and research today are more concentrated on the FTTx system, which provides the optical line to the each house. The limitations on the FTTx system are that the components and equipment used may incur a high cost of implementation.

In the existing multiplexer system, the technique used to increase the bandwidth is TDM technique. The conventional method is by using the electrical signal, which uses a coaxial or twisted pair cable. The optical solution available requires a pair of fiber for a single connection to the end user. The connection is made from the exchange point to the customer premises and fixed at certain data rate only. Inflexibility of existing solution pushes the telecommunication company using existing E1 architecture and increases the availability of exchange point to the place where it does not cover before. As the number of users increases the number of port has to be increased and introduce complexity in network. It also raises the price since it needs a number of ports in order to reach many users.

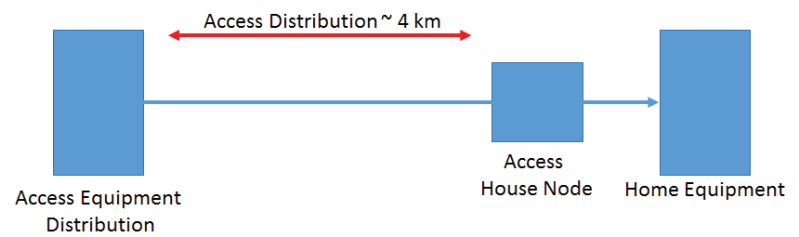

Fig. 1. Existing network architecture in access distribution node

The proposed multiplexer uses the CWDM technique to multiplex the optical signal into a single connection, thus increasing the capacity and optimizing the fiber usage. The interfacing of electrical signal is done at the media converter where it converts signals to optical signals independent of data rates and data formats and then multiplexed to the network optically. The signals received by the end user are demultiplexed according to the specific channel and are converted to electrical signal. The end user equipment may consist of a media converter only or a multiplexer depending on the service used. The system also supports electrical multiplexing such as in the fiber multiplexer. The multiplexed electrical signal is converted using media converter before transmitting into the proposed multiplexer. Figure 2 below shows the implementation of multiplexed access network into the access network today where the distribution lines from access network as shown in Figure 1 is converted into architecture in Figure 2.

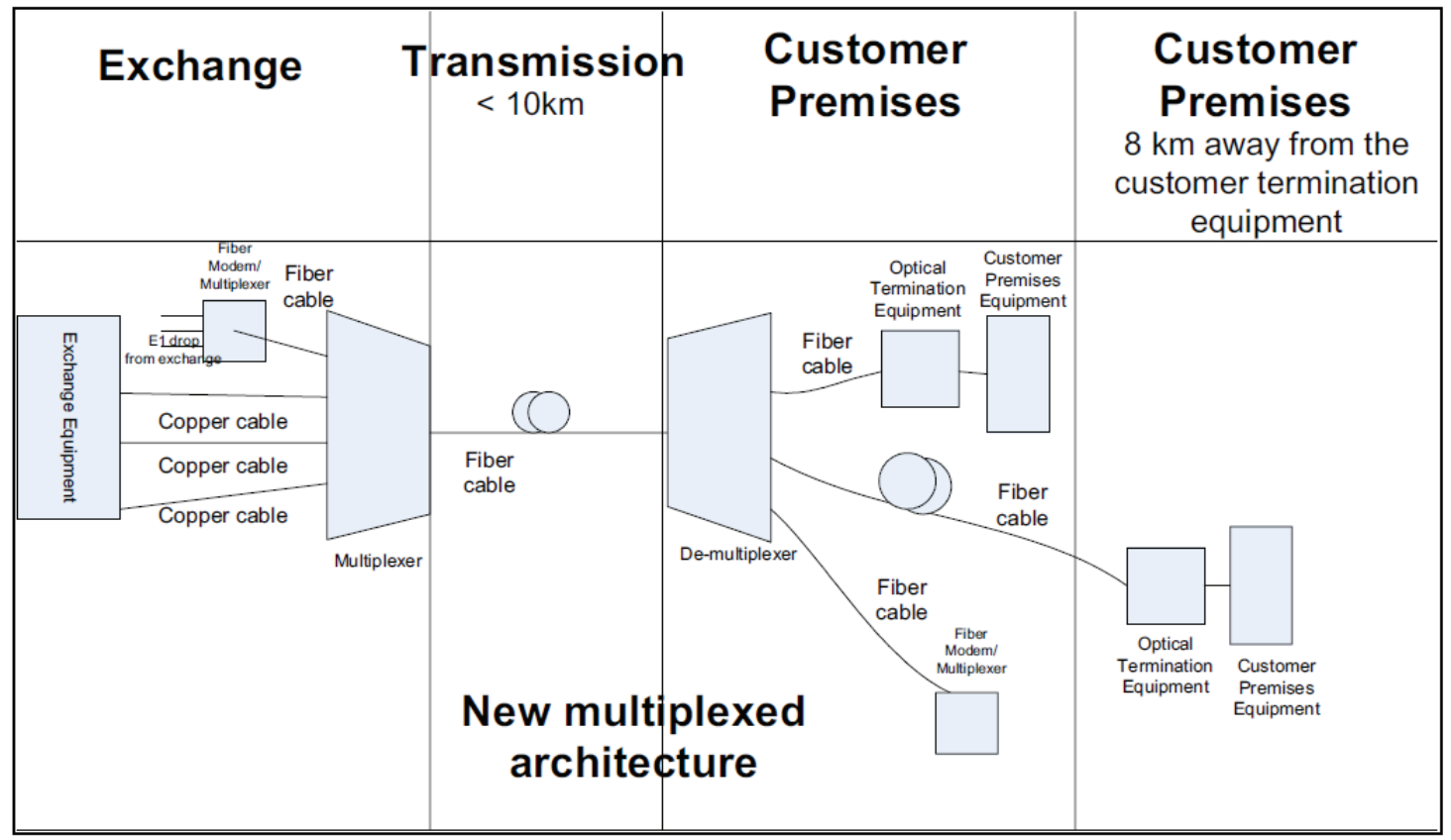

Fig. 2. Multiplexed access network architecture. 
The architecture of this system is expandable architecture where it can be expanded to nearby customers by optical or electrical connection depending on the distance of the other users as shown in Figure 2. The existing point-to-point architecture has been changed to the bus architecture in the new design. The new architecture provides the transmission media with either optical or electrical or both. This architecture is capable of supporting existing equipment used in the old system (existing architecture) either optical devices or electrical devices. The utilization of fiber in the network also can be increased since more than one signal can be transmitted in the same fiber. The nearby users can be connected easily by extending the network. The existing limitation faced by the previous network such as distance reach, limited number of users, cost incurred as the number of user increases, and electrical problems, can be eliminated by implementing this new network architecture.

In this architecture, the distance reachable problem is solved by extending the distance using optical cables. The connection to the end user can also be maintained in electrical domain since the proposed multiplexer supported both (optical and electrical) type of signals. The number of user can be easily added by using the fiber multiplexer or single connection of E1 services to the nearby users. Adding the number of users does not require new installation of new fiber. The usage of fiber can be maximized since all connection will be multiplexed into a fiber. This indirectly reduces the cost incurred in the transmission. The electrical problem is not a main issue in the proposed multiplexer. If the electrical problem arises at a certain user, it will not disturb other users in the network. The electrical problem of the system is also reduced since the proposed multiplexer is mainly in optical domain where it is immune to the electrical problems.

The architecture proposed is not only capable to multiplex all optical signals but also electrical signals. The multiplexer has an electrical port for all electrical termination in the access network. The converter has design where it can receive all type electrical formats. The special feature on this multiplexer is it can convert all electrical signal with format independent up to 600 Mbps. This will be able all electrical signal including the 2 wire and 4 wire format for telephony system that can be converted to electrical signal. Thus, all networks at distribution access node can be combined into a single network until up to end user or to the curb. This technique will reduce the complexity in the existing network and also cost of implementation.

\section{Results and Analysis}

Existing techniques that convert the electrical into optical depends on the format of the signal such as fiber multiplexer, media converter and EO converter. For example, fiber multiplexer only converts the E1 electrical signal and media converter converts the Ethernet signals. Furthermore, the price of individual equipment is considered expensive compare the purpose's multiplexer. The comparison of price is shown in Table 1. As clearly shown in the Table 1, the price of individual equipment for the conventional converter only is about same as complete proposed multiplexer. It clearly shows that the proposed multiplexer is considered economical compared to the conventional system since it provides better flexibility and new features to the network architecture and also competitive prices.

Table 1. Price comparison between conventional equipment and proposed system.

\begin{tabular}{|l|l|}
\hline $\begin{array}{l}\text { Proposed Multiplexer } \\
\begin{array}{l}\text { Converter RM 2,000 (Development } \\
\text { price not a sale price) }\end{array}\end{array}$ & EO Convertor RM 5,000 [4] \\
\hline $\begin{array}{l}\text { Multiplexer RM 2, 000 for 2 channel } \\
\text { (Development price not a sale price) }\end{array}$ & Media Convertor RM 2,220 [5] \\
& Fiber Multiplexer RM 8,000 [6] \\
& $\begin{array}{l}\text { Array wave guide/ Multiplexer RM 15,000 } \\
{[7]}\end{array}$ \\
\end{tabular}

The experiment setup to analyze the performance of the new CWDM system is shown in Figure 3.

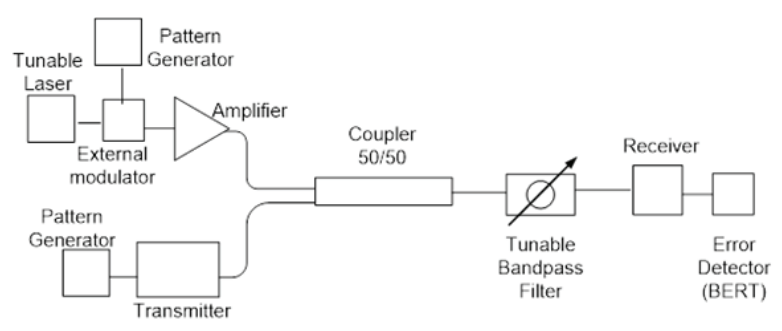

Fig. 3. Experimental setup to test the proposed system in access network.

This setup is a point-to-point transmission where the distance of the transmission is based on power budget calculation. The main blocks of the experiment are the transmitter, coupler, tuneable band-pass filter and the receiver. Coupler acts as the multiplexer while the tuneable bandpass filter acts as the de-multiplexer.

The main signal is modulated by the external modulator, while the adjacent signal is modulated from the $2.5 \mathrm{Gbps}$ transmitter. The main signal has to be amplified since the external modulator attenuates the transmitted power by $8.3 \mathrm{~dB}$. Both signals are then, multiplexed by coupler before being filtered by the tuneable bandpass filter to recover back the main signal. The performance of this setup is determined by the BER value detected by BER tester at the receiving side. The BER calculation is based on the $95 \%$ confidence level at $1 \mathrm{e}^{-12}$ error rates with 2 minutes requirement for every data collected. The CWDM portion in the architecture is tested for various data rate from $1.2 \mathrm{Gbps}$ up to 2.5 Gbps. The system is tested on the wavelength spacing variation towards the performance of the signal. It found that the BER is better when the wavelength spacing 0.58 
$\mathrm{nm}$. Figure 4 shows the performance of the system in term of BER towards variation spacing.

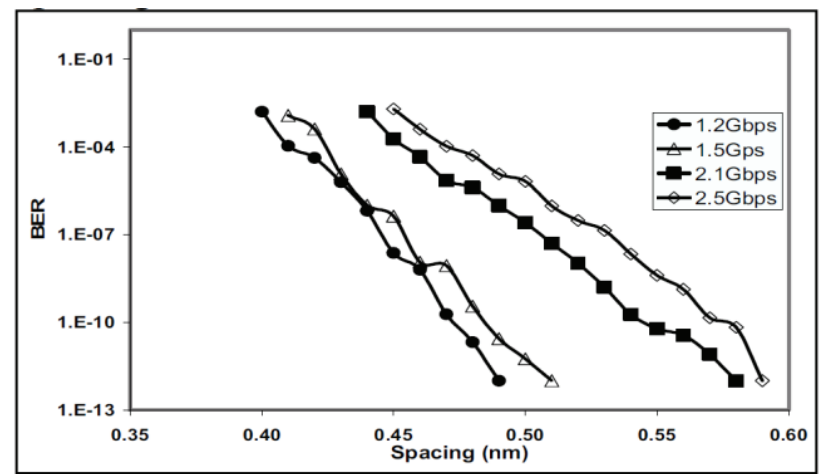

Fig. 4. The BER of system modulated adjacent signal performance towards wavelength spacing at different data rate.

The graph also shows that the wavelength-spacing can be reduced more when the data rate transmitted is reduced. This shows that the architecture can provide a better performance even though the transmitted signal is at 2.5 Gbps. The graph shows that decreasing the wavelength spacing will increase the system BER. When the spacing reduces, the overlapping spectra between channels increases. This will increase the cross-talk and introduce error into the channel, thus increasing the BER. It is important that to find the optimize spacing since it provide the number of channel can be transmitted in single core while reducing the price of services.

The system is also tested on the coverage span as shown in Figure 5. The system shows a better performance even though the data rate transmitted at 2.5 Gbps. It is found that the system can reach up to $15.4 \mathrm{~km}$ distance when system at $2.5 \mathrm{Gbps}$. As the data rate decreased, the graph shifted to the right, towards higher attenuation indicating that the system's sensitivity is better (higher) at lower data rate. For example, the system could attenuate up to $2.1 \mathrm{~dB}$ only for a stable performance at $2.5 \mathrm{Gbps}$ system but the system could attenuate up to $4.2 \mathrm{~dB}$ when the data rate was $155 \mathrm{Mbps}$.

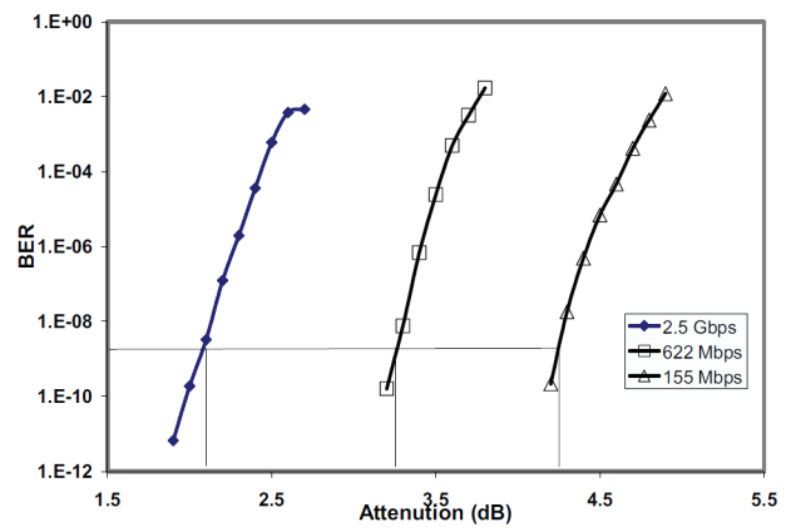

Fig. 5. BER at different data rate as attenuation increased.

Since this is a WDM system, in order to transmit higher data rates and longer distances, each channel can just carry $155 \mathrm{Mbps}$ and can be multiplexed to obtain higher bandwidth such as $622 \mathrm{Mbps}$ or $2.5 \mathrm{Gbps}$ whereas transmitting the data of $2.5 \mathrm{Gbps}$ in a single channel, will only support a short transmission distance. Thus, the system can support higher bandwidth with multiple channels. The system can reach up to $21.5 \mathrm{~km}$ at $155 \mathrm{Mbps}$ data rate. This bandwidth is already sufficient to support the needs at the end user.

\section{Conclusions}

The implementation of the CWDM in the access will resolve the current problem arises today especially on the bandwidth and distance reach. The architecture can be used as an intermediate solution before implementing full optical lines to the home. It was found that the system can transmit each channel up to $2.5 \mathrm{Gbps}$ with the probability of error of $10^{-12}$. The maximum distance that can be supported by this system is about $27 \mathrm{~km}$, for the data rate of $155 \mathrm{Mbps}$.

The author would like to acknowledge this work was supported by the High Ministry of Education Malaysia. The work has been done in 2003. The author would like to thanks to Late Prof Dr Mohamed Khazani Abdullah and his team at Universiti Putra Malaysia previously in 2003. Most of the work was done in Universiti Putra Malaysia previously.

\section{References}

1. R. Nelson., 2003, .Fibre in the access network where are the opportunities in Asia?.,http://www.ovum.com/Fibre in theaccess network - where are the opportunities in Asia.htm

2. Q. Yaojun, Q. Jiang, P. Hongtu, C. Shuqiang, G. Kejian, WCC. ICCT 2000 Communication Technology Proceedings, 2, pp. 1201 - 1204 (2000)

3. A.A.M. Saleh, J. M. Simmons, Journal of Lightwave Technology, 17, Issue 12, pp. 2431 2448 (1999)

4. Nvision, SD 4171 SDI E/O Converter. http://www.nvision1.com/Prod

5. Omnitron iConverter GX/T -media converter,http://reviews.cnet.com/Omnitron_iC onverter_GX_T_media_converter/4505243_7306435 $\overline{5} 4 . \overline{\mathrm{html}}$

6. RDMux 200. Fiber Multiplexer, http://www.raddirect.com/Product-FiberMultiplexer-RDmux200.html

7. JDSU Filter, $100 \mathrm{GHz}$, Add/Drop Module, http://www.jdsu.com/index.cfm?productid=280 \&pagepa th $=$ Products. 